\title{
Antimicrobials in Dentistry: With Knowledge Comes Responsibility
}

\author{
Walter J. Loesche
}

I $\mathrm{n}$ a recent editorial, Jørgen Slots and Thomas Pallasch have commented on the dentist's role in halting the development of antimicrobial resistance among bacterial species. The spread of antimicrobial-resistant micro-organisms among human and animal hosts has been the subject of much concern among physicians and discussion in the scientific and lay literature (Garrett, 1994). It is very appropriate that this issue be addressed from a dental perspective.

Slots and Pallasch have carefully reviewed the current usage of systemic antimicrobials in dental practice and describe two situations where systemic antimicrobials would be used for treatment purposes, and two situations in which a prophylactic usage would seem to be recommended. The treatment indications apply to the treatment of acute orofacial infections with systemic manifestations, and to the treatment of certain types of severe periodontitis and peri-implantitis. The prophylactic indications would apply to medical conditions such as endocarditis and for the "prevention of traumatic/infectious sequelae to surgical dental procedures". They then qualify these prophylactic situations to eliminate any ambiguities. For example, they provide a comprehensive listing of medical conditions for which antimicrobial prophylactics would and would not be recommended during dental treatment. They review those dental surgical procedures where prophylactic antibiotics might be indicated, and conclude that "the only dental treatment that might qualify would be the placement of dental implants".

The usage of systemic antimicrobials for prophylactic purposes in dentistry is rarely based upon documented clinical data. Even in the well-accepted usage of prophylactic antimicrobials in individuals with endocarditis, the estimated rate of protection from an infectious episode is balanced by the estimated rate of death from an untoward reaction to the systemic antimicrobial. Yet, for mainly legal and historical purposes, this practice has become the de facto standard of care.

There is even less support for the routine use of systemic antimicrobials prophylactically following oral and/or periodontal surgery. The prevalence of post-operative infections following periodontal surgery is less than one percent, and this low risk would not justify the prophylactic usage of systemic antimicrobials. Yet according to American Academy of Periodontology surveys conducted in 1981 and
1987, about two-thirds of the respondents reported using mainly tetracyclines and penicillins for just this purpose. This usage would seem unwarranted and is contrary to the recommendation of the Medical Letter that antibiotic prophylaxis should be used only for surgical procedures with a high infection rate.

Generally speaking, the dentist has not overused or misused antibiotics in treatment situations. This has a historical basis, since the prevailing 19 th century treatment paradigm was based on the assumption that dental decay and periodontal disease were due to this non-specific overgrowth of plaque bacteria, i.e., the "non-specific plaque" hypothesis. This paradigm essentially dismissed any suggestion of the use of chemical antimicrobial agents, because they were unsafe and unnecessary, given the alternative of physical debridement of the tooth by mechanical means. In that context, the non-use or minimal usage of systemic antimicrobials was appropriate and helped keep the dentist from the abusive usage of antimicrobials.

But as we enter the 21 st century, a new treatment paradigm has appeared which indicates that both dental decay and periodontal disease are specific, albeit chronic, bacterial infections. Support for the specific-plaque hypothesis rests upon epidemiological evidence that while decay and periodontal disease are ubiquitous in the population, the severe forms are clustered in small subsets of individuals. When plaques from these individuals are examined bacteriologically, plaques from diseased sites or from patients with high dental morbidities have elevated levels and/or proportions of certain bacterial species when compared with plaques taken from non-diseased tooth sites or from dentally healthy individuals.

The unequivocal demonstration of an etiologic role for specific organisms in chronic mucous membrane infections is fraught with difficulties. This is even more so when the putative odontopathogens - such as Streptococcus mutans, Lactobacilli casei, Treponema denticola, Bacteroides forsythus, Porphyromonas gingivalis, and Prevotella intermedia, among others - are commonly found in plaque and saliva samples of dentally healthy individuals. Because dental decay and periodontal disease behave as endogenous infections, this rules out any diagnostic significance associated with the mere presence of these organisms. It is necessary to demonstrate elevated levels and proportions of these species 
before any diagnostic implications can be made from bacteriological testing. Such quantitative bacteriological tests are not readily available, and those that exist are both underutilized and minimally documented. This is of immediate concern, because if antimicrobials are to be used in dental therapies, they will need to be focused toward the suppression or elimination of specific bacterial species in the disease-associated plaques.

Once the concept of infection is introduced for dental decay and periodontal disease, then the assumption is that all reasonable means to treat these infections, including the prescription usage of systemic antimicrobials, will be considered. This is a departure from traditional dental therapy and comes at a time when most health authorities are urging caution in the usage of systemic antimicrobials. If there will be a testing ground for the relative merits of the antimicrobial approach to treatment $v$ s. the traditional surgical-debridement approach to treatment, it will be in periodontal therapy.

The success of agents such as metronidazole, clindamycin, and doxycycline in refractory periodontitis begs the question as to how successful these agents would be if introduced earlier in the treatment process, prior to any surgical intervention. There is general agreement that the surgical approach is successful in about $80 \%$ of advanced cases but, because of its cost and general unpleasantness, is not wellaccepted by the public. More people are retaining their teeth into old age and therefore will be at risk to developing periodontal disease. Because of this, and because periodontal disease is being suggested as a risk factor for coronary heart disease and stroke, there is a need to reconsider how we treat advanced forms of periodontal disease.

We have conducted three double-blind clinical studies in which systemic metronidazole in conjunction with mechanical debridement (scaling and root planing) was shown to reduce the need for access surgery. We selected patients in whom access surgery and extractions were deemed necessary according to traditional criteria. We then showed that in over $90 \%$ of these patients, an anaerobic infection - defined as a predominance of anaerobic bacteria such as spirochetes, $T$. denticola, $P$. gingivalis, and B. forsythus - was present in four plaque samples removed from sites with deep pocketing and bone loss. The combination of clinical disease plus the demonstration of anaerobes led us to choose metronidazole, an antimicrobial agent that is specific for Gram-negative anaerobes. We used rigorous debridement to reduce the bacterial loads on the tooth surface non-specifically, and then followed with a dosage of metronidazole, adjusted for the patient's body weight, for a one- or two-week period. Because compliance with metronidazole may not be good, we evaluated doxycycline and found this agent also to be effective in reducing surgical needs.

In each of these studies, the combination of debridement plus one or two weeks of unsupervised usage of systemic metronidazole reduced the need for surgery, compared with the debridement plus placebo control. In the third study, we were able to show that by the administration of up to five rounds of either systemic or locally delivered antimicrobials, about $90 \%$ of the teeth initially scheduled for surgery, or in some cases extractions, could be spared from surgery or extractions. Only $20 \%$ of the patients required some form of surgery, and then at a reduced level. This is comparable with the $20 \%$ of patients who are refractory to traditional therapy and need systemic antimicrobials.

These results suggest that patients and clinicians now have a choice in the treatment of advanced forms of periodontal disease: the traditional surgical approach with systemic antimicrobials held in reserve for refractory cases, or the infection approach with surgery held in reserve for refractory cases (teeth). In both treatment approaches, the need for the diagnosis of infection and the choice of antimicrobial agent is important. This is a point emphasized by Slots and Pallasch and cannot be ignored.

As promising as these results appear, there is a concern that that they could be trivialized by the thinking that antimicrobials are a panacea for all forms of periodontal disease, and possibly could be used prophylactically to prevent individuals from getting periodontal disease. Antimicrobials are powerful agents when used in wellfocused ways. We carefully selected patients who had advanced forms of periodontitis, and in whom access surgery would be indicated according to prevailing treatment practices. Because we were able to document an anaerobic infection, we were able to select the appropriate agent. This approach and results cannot be directly transferred to forms of moderate periodontitis or to gingivitis. These clinical entities respond to mechanical debridement, and both risk-benefit and cost-benefit considerations would not seem adequate to justify the usage of antimicrobial agents.

We are at a point in dentistry where clinical dividends can be achieved from the many years of research which have shown that most forms of dental decay and periodontal disease are diagnosable and treatable infections. We are also in a transition process from a health profession which relied exclusively upon a surgical approach to combat the ravages of dental decay and tooth loss, to one in which a combined surgical and medical approach will be needed to maintain an individual's optimal dental health for a lifetime. We will need to recognize those individuals at risk to clinical infections and to be able to treat them cautiously. The alarms being raised about the overuse and misuse of systemic antimicrobials need to be heeded so that we do not as a profession contribute to this "coming plague". We will need to add the knowledge of an infectious disease specialist to our surgical skills.

\section{Additional Reading}

Garrett L (1994).The coming plague. New York, NY: Penguin Books, $750 \mathrm{pp}$

Loesche WJ, Giordano J, Sohren S, Hutchinson R, Rau CF, Walsh L, et al. (1996). Nonsurgical treatment of patients with periodontal disease. Oral Surg Oral Med Oral Pathol 81:533-543.

Dr. Loesche is Professor of Dentistry, School of Dentistry, and Professor of Microbiology and Immunology, School of Medicine, University of Michigan, Ann Arbor MI 48109. 